STRUCTURAL BIOLOGY

ISSN 2059-7983

\section{Iron-Sulfur Clusters in Chemistry and Biology. Volume 1: Characterization, Properties and Applications. Second edition. Edited by Tracey Rouault. De Gruyter, 2017. Pp. xv + 447. Price EUR 99.95, GBP 90.99, USD 114.99 hardcover, ISBN 978-3-11- 047850-1.}

\author{
Jean-Pierre Jacquot*
}

Université de Lorraine, INRA, Laboratoire Interaction Arbres Microorganismes, Nancy, France. *Correspondence e-mail: j2p@univ-lorraine.fr

Volume 1 of the second edition of this book dedicated to iron-sulfur clusters and edited by Tracy Rouault, deals with characterization, properties and applications. Compared with the earlier edition coordinated by the same editor and also published by de Gruyter there are several changes, the most noticeable being that the book has now been split into two different volumes. While I have not yet had the chance to look into the second volume, I have compared this new first volume to the earlier edition. The content has been split in such a way that this first volume deals primarily with physico-chemical properties of iron-sulfur centres and also describes in a very chemistry-oriented way the biochemical mechanisms of a selected number of enzymes (among them biotin synthase, nitrogenase and a number of molybdenum-containing enzymes). Clearly all chapters dealing with the assembly of iron-sulfur clusters have been moved to Volume 2 and hence the reader should not expect much information about this fast developing topic in Volume 1. Compared to the first edition this volume now contains three additional chapters by Noodleman; Guo and Li; and Dodd, Crack, Thomson and Lebrun. The Noodleman chapter deals with quantum chemistry and is possibly a bit redundant but also complementary to an earlier chapter by Ichiye (also present in the new edition and describing the chemical properties of iron-sulfur clusters). The Dodd et al. chapter deals with the reactivity of iron-sulfur clusters with nitric oxide, a most welcome addition in a relatively new field. The other chapters found in this new volume were present in the earlier edition and they include a historical perspective (Bonomi and Rouault), and three chapters discussing various spectroscopic methods for the characterization of ISCs (Guo and Li, Petasis and Hendrich, Chakrabarti and Lindahl). Two additional chapters describe nitrogenase and the discovery of its interstitial carbide from two different perspectives (Sickerman et al., Spatzman et al.). The radical mediated generation of carbon-sulfur bonds is addressed in a chapter on biotin synthase by Jarrett; Hille describes molybdenum-containing enzymes other than nitrogenase; Lanz Squire and Booker detail the role of iron-sulfur clusters in biosynthesis of the lipoyl cofactor; and finally Nicolet and Fontecilla Camps discuss the relationships between ISCs and oxygen. Overall there are thus 13 chapters in this volume. In general, the perspective depends very much on physics and chemistry and thus it is a lot easier to read for physico-chemists than for biologists. It is expected that the second volume should be a lot easier to digest for biologists. Still it is worth the effort of jumping into the orbitals and spins to truly understand the meaning of the EPR Raman and Mössbauer spectra, and in this respect this volume is certainly a lot more detailed than the previous edition. 